Methods Adult patients were recruited from specialist bronchiectasis and Cystic Fibrosis clinics. The gold standard for diagnosing $P$. aeruginosa infection was positive sputum cultures. 72 sputum samples were analysed. A sputum sample was kept in a glass vial with a cap containing septum. The septum was pierced with a solid phase microextraction (SPME) fibre allowing sampling of the headspace for $50 \mathrm{~min}$ at $37^{\circ} \mathrm{C}$ before transferring the fibre into gas chromatography mass spectrometry. AnalyzerPro software (automated peak capture software) and manual identification were used to identify relevant to $P$. aeruginosa specific compounds in the headspace of sputum.

Results 32 samples grew $P$. aeruginosa either on its own or mixed with other species. 2-nonanone was a marker of $P$. aeruginosa in sputum headspace gas with sensitivity of $72 \%$ and specificity of $88 \%$. Cyanide was not detected. However, a combination of manually identified 2-nonanone with 17 other volatile compounds as identified by AnalyzerPro, increased sensitivity in detection of $P$. aeruginosa to $91 \%$ with specificity of $88 \%$.

Conclusion Optimal sampling and capture protocols still need refinement: we were unable to detect the prior noted biomarker Cyanide. These data however demonstrate the potential for rapid and accurate diagnosis of $P$. aeruginosa infection from sputum samples. In contrast to the $48+$ hour turnaround for standard microbiological culture, these results were available within $1-2 \mathrm{~h}$. It also provides a library of compounds as targets to validate in a future study of breath testing.

\section{S22 LUNG CLEARANCE INDEX, FEV1 AND CT FINDINGS IN CYSTIC FIBROSIS: DATA FROM THE UK CF GENE THERAPY CONSORTIUM RUN-IN STUDY}

doi:10.1136/thx.2010.150912.22

${ }^{1} \mathrm{H}$ S Sheridan, ${ }^{2} \mathrm{~N}$ J Bell, ${ }^{1} \mathrm{~K}$ A Macleod, ${ }^{2} \mathrm{P}$ A Reid, ${ }^{3} \mathrm{~A}$ R Horsley, ${ }^{4} \mathrm{G}$ Davies, ${ }^{4} \mathrm{C}$ Saunders, ${ }^{1} \mathrm{~S}$ Cunningham, ${ }^{2} \mathrm{~J} \mathrm{~A}$ Innes, ${ }^{4} \mathrm{~J} \mathrm{C}$ Davies, ${ }^{3} \mathrm{E}$ W Alton. ${ }^{1}$ The Royal Hospital for Sick Children, Edinburgh, UK; ${ }^{2}$ Western General Hospital, Edinburgh, UK; ${ }^{3}$ UK CF Gene Therapy Consortium, UK; ${ }^{4}$ Department of Gene Therapy, Imperial College, London, UK

Introduction Lung Clearance Index (LCI) is a measure of lung gas mixing derived from the Multiple Breath Washout (MBW) test. We present LCI, FEV 1 and CT data from the Run-In Study, a longitudinal study in preparation for a multi-dose trial of nebulised gene therapy for CF.

Methods MBW, spirometry and low-dose HRCT chest were performed as part of the first Run-In Study visit. LCI was reported as the mean result from at least two technically acceptable sulphur hexafluoride MBW tests performed using a modified Innocor gas analyser. Spirometry was performed to ERS standards. CT scans were assessed by two independent radiologists for extent and severity of bronchiectasis, wall thickening, presence of small and large airway plugs, and gas trapping.

Results 191 patients attended visit 1, mean (range) age 22.6 (10-59.1) years. Validated LCI, FEV 1 and CT results were available for 167, 191 and 150 patients, respectively. Mean (SD) FEV 1 was 72 (19)\% predicted. Mean (SD) LCI was 10.7 (2.7), with mean intravisit coefficient of variation of $4.9 \%$. LCI correlated negatively with $\mathrm{FEV}_{1}$ $(r=-0.68, p<0.001)$, but was abnormally elevated in $72 \%$ of participants with normal $\mathrm{FEV}_{1}$ (see Abstract S22 Figure 1; triangles indicate $\mathrm{FEV}_{1}>80 \%$ and LCI $>7.5$ ). $95 \%$ CI for LCI in normal subjects 5.9 to 7.5. Both $\mathrm{FEV}_{1}$ and LCI correlated with all CT measures $(p<0.001)$, most strongly with extent of bronchiectasis. LCI correlated better than $\mathrm{FEV}_{1}$ with extent of bronchiectasis, $r=0.72(p<0.001)$ vs $r=-0.61(p<0.001)$, respectively.

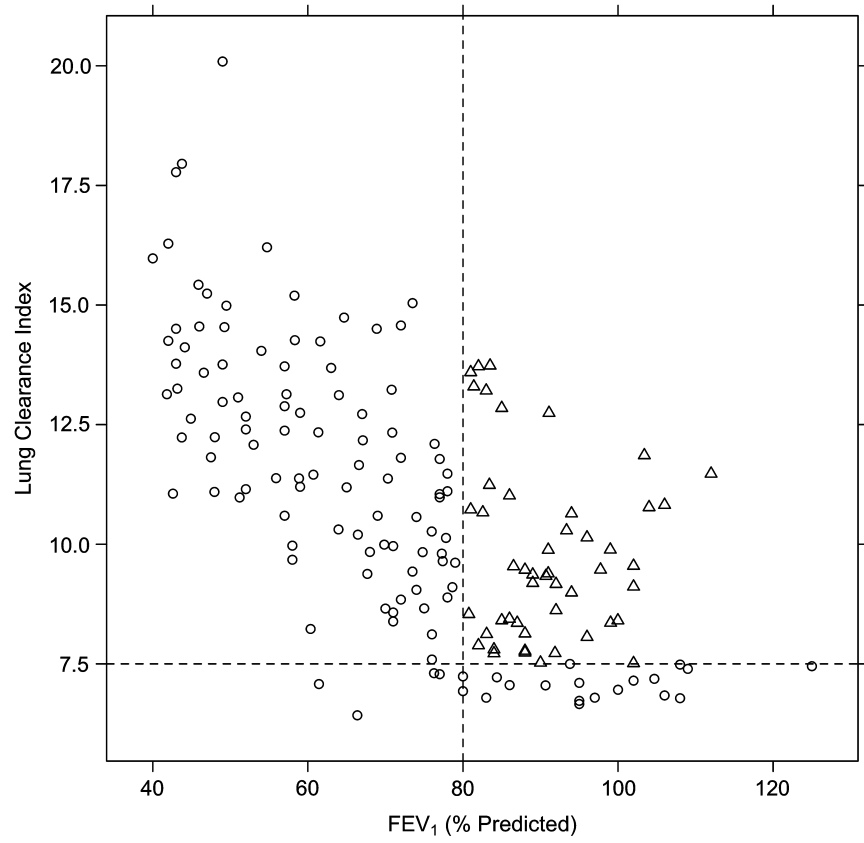

Abstract S22 Figure 1

Conclusions Results from this large cohort suggests that LCI is a more sensitive test of early CF lung disease, and correlates better with extent of bronchiectasis seen on CT, than $\mathrm{FEV}_{1}$. Validation of data from subsequent study visits is in progress and will be reported at a future date.

\section{S23 A COMPARATIVE STUDY OF POLYMICROBIAL DIVERSITY IN CF AND NON-CF BRONCHIECTASIS}

doi:10.1136/thx.2010.150912.23

${ }^{1} S$ P Cummings, ${ }^{1} A$ Nelson, ${ }^{1} \mathrm{P}$ J Purcell, ${ }^{2} \mathrm{~A}$ De Soyza, ${ }^{3} \mathrm{~S} J$ Bourke, ${ }^{4} \mathrm{~J} D$ Perry. ${ }^{1}$ School of Applied Sciences, Ellison Building, University of Northumbria, Newcastle upon Tyne NE1 8ST, UK; ${ }^{2}$ Lung Transplantation and Immunobiology Group, Newcastle University and the Freeman Hospital Newcastle upon Tyne, UK; ${ }^{3}$ Adult Cystic Fibrosis Unit, Department of Respiratory Medicine, Royal Victoria Hospital, Newcastle upon Tyne, UK; ${ }^{4}$ Department of Microbiology, Freeman Hospital, Newcastle upon Tyne, UK

Introduction and Objectives Bronchiectasis is a dilation of the peripheral airways with subsequent mucus hypersecretion. Bronchiectasis can be either genetic, that is cystic fibrosis (CF) or described as non-CF bronchiectasis (eg, idiopathic or post infectious bronchiectasis). Recently, many studies have demonstrated polymicrobial bacterial communities are present in the lower respiratory tract (LRT) of cystic fibrosis (CF) sufferers. These studies have identified complex microbial communities that are affected by many factors including age; CFTR genotype and antibiotic therapy. One prior abstract noted greater diversity in non-CF bronchiectasis as compared to CF (Bilton et al, 2009) though the sample size was small. Our aim is to extend prior work by comparing the metabolically active bacterial and fungal communities present in sputum samples from CF patients with those from non-CF bronchiectasis. Methods Adult CF and non-CF bronchiectasis patients provided spontaneously expectorated sputum samples which were treated with RNAlater. RNA was extracted from sputum samples and reverse transcribed to cDNA; this was the template for bacterial and fungal community PCR amplification using universal $16 \mathrm{~S}$ or $28 \mathrm{~S}$ primer sets. Amplicons were analysed by denaturing gradient gel electrophoresis (DGGE) which separates double stranded DNA based upon bacterial and fungal genomic GC content sequence. Common pathogens were identified such as Pseudomonas aeruginosa and Haemophilus spp. by comparison to a $16 \mathrm{~S}$ or $28 \mathrm{~S}$ standard ladder from pure cultures. 
Results We have compared CF and non-CF bronchiectasis ( $\mathrm{n}=36$ combined). Polymicrobial communities were observed in all CF and non-CF bronchiectasis patients. However, CF patients demonstrated a greater bacterial diversity with a mean of 14.77 species per sample (range 6-21) than non-CF bronchiectasis patients who had a mean of 9.67 species per sample (range 4-14). However, fungal communities were similar between CF and non-CF bronchiectasis with $73.3 \%$ and $75 \%$ of patients harbouring fungi in their LRT respectively. Similarly, CF patients had a mean of 1.33 fungal species per sample (range 0-4) whilst non-CF bronchiectasis patients had a mean of 1.16 fungal species per sample (range 0-3).

Conclusions We note a complex microbiota in the lungs of both CF and non-CF bronchiectasis patients. In contrast to other studies using DNA based molecular analysis we note an increased microbial diversity observed in the CF cohort. The increases in bacterial taxa in CF may be due to differences in CFTR status, disease duration, or the intensive antibiotic regimens creating differing biological niches in non-CF bronchiectasis.

\section{Paediatric infectious diseases S24 DIFFERENTIATED PRIMARY BRONCHIAL EPITHELIAL CELL (PBECS), MONOCYTE DERIVED MACROPHAGES (MDMS) AND MONOCYTE DERIVED DENDRITIC CELLS (MODCS) TRANSWELL CO-CULTURE: RESPIRATORY SYNCYTIAL VIRUS (RSV) INFECTION OF THE APICAL AND BASOLATERAL SURFACES}

doi:10.1136/thx.2010.150912.24

\section{K B Ugonna, K Plant, M L Everard. University of Sheffield, Sheffield, UK}

Introduction and Objectives RSV causes winter epidemics of respiratory disease. Active infection is virtually absent in summer months. Infected ciliated airway epithelial cells, local macrophages and dendritic cells secrete cytokines including interleukins (IL) 6 and 8, promoting a strong neutrophilic response that is important in disease clearance and airway inflammation. In vitro, RSV is capable of infecting MoDCs. RSV inhibits their maturation and can remain dormant in these cells. Dormant RSV can be stimulated to replicate with exogenous nitric oxide. These MoDCs are then able to re-infect HeLa cells (a lab strain of immortalised cervical cells). The following hypothesis was explored: Infected dendritic cells act as a reservoir for $R S V$ over summer months.

Aim The aim of this study was to investigate the effect of RSV on $\mathrm{pBEC}$ and MoDc cell lines across a semi permeable membrane in the presence of MdMs.

Methods Primary bronchial epithelial cells were seeded in the apical part of the transwell model at $1 \times 10^{6}$ cells per $\mathrm{ml}$ and differentiated over 21 days on an air liquid interface. MoDCs were seeded on the basolateral part of the transwells at $1 \times 10^{6}$ cells $/ \mathrm{ml}$. MdM were seeded on top of the epithelial layer in selected experiments (see Abstract S24 Figure 1). Red fluorescent RSV (rrRSV) was added to the apical side at a concentration of $1 \times 10^{6}$ plaque forming units (pfu)/ml with un-infected MoDCs on the basolateral side, and uninfected pBECs in the apical side were cocultured with MoDCs previously infected with rr-RSV at $5 \times$ $10^{5} \mathrm{pfu} / \mathrm{ml}$. Controls were uninfected pBECs with uninfected MoDCs or with just media on basolateral side. Red fluorescence (marker for active infection) was measured at 24, 48 and $168 \mathrm{~h}$ by flow cytometry.

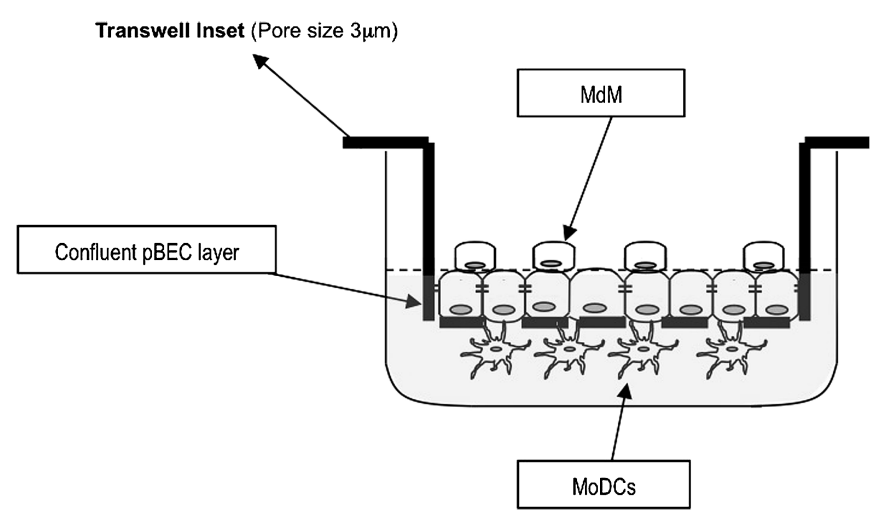

Abstract S24 Figure 1

Results Directly exposed pBECs and MoDCs were infected at $24 \mathrm{~h}$. Indirectly exposed pBECs were infected at $48 \mathrm{~h}$. Indirectly exposed MoDCs were infected only when MdMs were present on the overlying epithelial cell layer.

Conclusions RSV is able to infect MoDCs and pBEC across a semipermeable membrane in our in vitro model of airway epithelium, supporting MoDCs as a potential summer reservoir of RSV.

\section{S25 IL 17 PRODUCTION IN PRIMARY AND SECONDARY RESPIRATORY SYNCYTIAL VIRUS (RSV) INFECTION AND NEUTROPHIL TRANSMIGRATION}

doi:10.1136/thx.2010.150912.25

K B Ugonna, K Plant, M L Everard. University of Sheffield, Sheffield, UK

Introduction and Objectives RSV causes winter epidemics of respiratory disease particularly in infants and the immunocompromised. Infected ciliated airway epithelial cells, local macrophages and dendritic cells secrete cytokines including interleukins (IL) 6 and 8, promoting a strong neutrophilic response that is important in disease clearance and airway inflammation. The IL 17 inflammatory pathway, which is important in surface immunity, has been little studied in RSV infection to date. The following hypothesis was explored: The IL17 pathway is important in neutrophil chemotaxis and restriction of $R S V$ replication.

Methods A transwell model of the airway was devised, co-culturing A549s (an immortalised bronchial epithelial cell line) and neutrophils to study the effect of RSV and IL17 on the transmigration of neutrophils. Neutrophil transmigration was assessed using light microscopic immunocytochemistry. Nasal swabs were taken from infants with RSV positive Bronchiolitis $(n=49)$, RSV negative Bronchiolitis $(n=12)$, the symptomatic older siblings of RSV positive infants $(n=15)$ and uninfected, asymptomatic children $(n=20)$. Cytokines released (IL6/IL8/IL17a/IL21/TNF) were analysed by cytokine bead array.

Results IL 17 and RSV caused significantly increased number of neutrophils to transmigrate compared to either IL 17 or RSV alone. Primary RSV infections which caused hospitalisation, are characterised by high levels of nasal epithelial derived cytokines (IL 6, IL 8). Less severe secondary RSV infections are characterised by relatively low levels of IL 6 and IL 8 and relatively high levels of IL 17.

Conclusions There is evidence that IL 17 is synergistic with RSV induced IL 8 in promoting neutrophil transmigration in vitro. There is evidence to support IL 17 as an important cytokine restricting RSV production in secondary infection in vivo. This study suggests that the IL 17 pathway is important in the pathogenesis of RSV bronchiolitis and could be potentially important in the development of novel therapies for this ubiquitous and important cause of respiratory disease. 